\title{
Origin and Bulk Chemical Composition of Mercury
}

\author{
Andrew J. R. Prentice and Daniel Jontof-Hutter \\ Mathematical Sciences, Monash University, Victoria 3800, Australia
}

\begin{abstract}
The origin of Mercury's high metal content is examined within a gas ring model for the condensation of the planetary system. Mercury's axial moment-of-inertia factor is predicted to be $0.325 \pm 0.002$.
\end{abstract}

The planet Mercury is remarkable because its mean uncompressed density $\sim 5.3 \mathrm{~g} / \mathrm{cm}^{3}$ implies a Fe-Ni mass percent content of $\sim 67 \%$. This is more than twice that of its neighbor Venus. This factor, coupled with other marked chemical differences between the four terrestrial planets, points to the conclusion that each planet 'received the overwhelming majority of its mass from narrow, compositionally-distinct annuli of material around the Sun' (Drake \& Righter 2002). This situation finds a natural explanation within the Modern Laplacian theory of Solar system origin [hereafter MLT] (Prentice 1978, 2001a, 2001b).

According to the MLT, the planetary system condensed from a concentric family of gas rings. Discrete ring shedding takes place through the action of a radial turbulent stress $\left\langle p_{t} v_{t}^{2}\right\rangle$ arising from powerful convective motions of speed $v_{t}$ within the proto-solar cloud [PSC] cloud. If $\left\langle\rho_{t} v_{t}^{2}\right\rangle$ equals $F_{t} \sim 35$ times the gas pressure $\rho v_{s}^{2} / \gamma$ at the PSC surface, where $\rho$ is the density, $v_{s}$ is the adiabatic sound speed and $\gamma=1.4$, then a steep density rise by a factor $\left(1+F_{t}\right)$ occurs and the orbital radii $R_{n}(n=0,1,2, \ldots)$ of the rings match the mean planetary distances. Such large values of $F_{t}$, however, imply $v_{t} \geq 5 v_{s}$, which is unlikely. To resolve this difficulty, Prentice \& Dyt (2003) conducted a numerical simulation of supersonic turbulent convection in a model atmosphere to mimic the upper layer of the PSC. They discovered that the introduction of a velocity-dependent thermal diffusivity $\kappa_{t}$ to model subgrid-scale motions induces a sharp negative temperature gradient at the top boundary. A modest density upturn factor of 3.5 ensues for peak vertical speeds $\left\langle v_{t}\right\rangle_{p} \simeq 1.1 v_{s}$. This implies that the MLT may be validated with values $\left\langle v_{t}\right\rangle_{p} \simeq 3 v_{s}$ and $F_{t} \simeq 10-15$, which are reasonable.

Consider now the results of a representative numerical simulation of the PSC's gravitational contraction which (i) accounts for the mean planetary distances, (ii) produces a Sun of the observed mass $M_{\odot}$, (iii) accounts for Mercury's high metal content and (iv) accounts for the 0.50:0.50 sub-solar ice-to-rock mass fractions of Ganymede and Callisto. In this simulation, the Mercurian gas ring is shed at orbital radius $R_{n}=76.9 R_{\odot}$, the residual cloud mass there is $M_{n}=1.082 M_{\odot}, T_{n}=1643 \mathrm{~K}$ and $p_{n}=0.1566$ bar.

A schematic view of the gas ring cast off at Mercury's initial orbit $R_{n}$ is given below. The ring is assumed to have a uniform temperature $T_{n}$ and orbital angular momentum per unit mass $\sqrt{G M_{n} R_{n}}$, where $G$ is the gravitation constant. The gas pressure at distance $\xi$ from the mean circular orbit is $p_{\text {gas }}(\xi)=p_{n} \exp \left(-\frac{1}{2} \alpha_{n} \xi^{2} / R_{n}^{2}\right)$, where $\alpha_{n}=\mu G M_{n} / \Re T_{n} R_{n} \simeq 467$ is a constant and $\mu=2.379$ is the mean molecular weight. Consider next the condensation of a 
chemical species $i$ whose vapor pressure is $p_{i \text {,vap }}=\exp \left(B_{i}-A_{i} / T_{n}\right)$. Here $A_{i}, B_{i}$ are thermodynamic constants - given below for reference temperature $1600 \mathrm{~K}$. Let $p_{i, n}(\xi)=\left(\mu / \mu_{i}\right) X_{i} p_{\text {gas }}$ denote the initial partial pressure, where $\mu_{i}$ and $X_{i}$ are the molecular weight and total mass fraction of the species. Condensation is then restricted to minor radii $\xi<\xi_{i}$ where $p_{i, n}(\xi)>p_{i, n}\left(\xi_{i}\right)=p_{i \text {,vap }}\left(T_{n}\right)$. The condensed mass fraction is $X_{i, \text { cond }}=X_{i}\left[1-\left(1+f_{i}\right) \exp \left(-f_{i}\right)\right]$, where $f_{i}=A_{i}\left(1 / T_{n}-1 / T_{i, n}\right)$ and $T_{i, n}=A_{i} /\left(B_{i}-\log \left(p_{i, n}(0)\right)\right.$ is the condensation temperature on the mean orbit $\xi=0$. For Fe $(i=1)$, we have $X_{1} / \mu_{1}=2.223 \times 10^{-5}$, $A_{1}=47670, B_{1}=16.089, T_{1, n}=1715 \mathrm{~K}$ and $X_{1, \text { cond }} / X_{1}=0.346$. For species 2 we choose $\mathrm{MgSiO}_{3}$. Here $X_{2}=\left(X_{\mathrm{H}_{2} \mathrm{O}} / X_{\mathrm{H}_{2}}\right) \sqrt{X_{\mathrm{Mg}} X_{\mathrm{SiO}}}=8.89 \times 10^{-6}$, $\mu_{2}=292.5, A_{2}=63773, B_{2}=20.759$, and hence $T_{2, n}=1633 \mathrm{~K}$. Since $T_{2, n}<T_{n}$, $X_{2, \text { cond }}=0$. Mercury thus condensed at such a high temperature that $\mathrm{MgSiO}_{3}$ (and also $\mathrm{Mg}_{2} \mathrm{SiO}_{4}$ ) was excluded from its bulk chemical makeup. Such an explanation for Mercury's low silicate content was first considered by Lewis (1972).
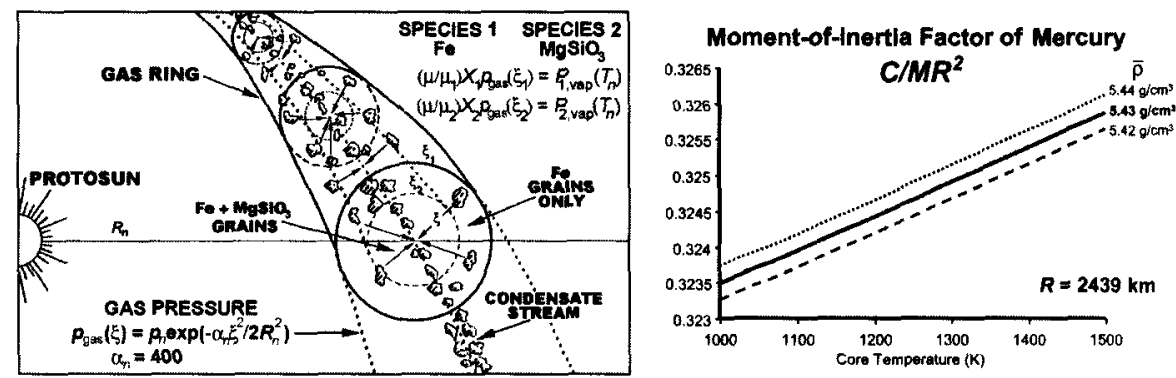

Figure 1. Left: A schematic view of the gas ring cast off at Mercury's initial orbit. Right: A plot of the moment-of-inertia factor vs $T_{c}$

The bulk composition (\& mass percents) of the Mercurian condensate are Fe-Ni-Cr-Co-V (67.00), $\mathrm{Ca}_{2} \mathrm{Al}_{2} \mathrm{SiO}_{7}$ (26.12), $\mathrm{MgAl}_{2} \mathrm{O}_{4}$ (4.07), $\mathrm{Al}_{2} \mathrm{O}_{3}$ (1.73) and $\mathrm{CaTiO}_{3}$ (1.08). A suite of simplified two-zone structural models based on this mix has been constructed. Each model has a Fe Ni core of mass $67 \%$ and uniform temperature $T_{c}$, and a gehlenite-spinel mantle of temperature $T_{m}$. Selfcompression is modeled with the properties of $\mathrm{Fe}$ and spinel. $T_{m}$ is chosen so that $\bar{\rho}$ matches the observed value $5.43 \pm 0.01 \mathrm{~g} / \mathrm{cm}^{3}$, subject to $700 \mathrm{~K} \leq T_{m} \leq$ $T_{c} \leq 1500 \mathrm{~K}$. A plot of the moment-of-inertia factor vs $T_{c}$ is shown. We predict that $C / M R^{2}=0.325 \pm 0.002$. This coincides with Siegfried \& Solomon (1974). We thank Charles Morgan and Steven Morton for technical assistance.

\section{References}

Drake, M.J.,\& Righter, K. 2002, Nature, 416, 39

Lewis, J.S. 1972, Earth Planet. Sci. Lett., 15, 286

Prentice, A. J. R. 1978, Moon \& Planets, 19, 341

Prentice, A. J. R. 2001a, Earth Moon \& Planets, 87, 11

Prentice, A. J. R. 2001b, http://www.lpi.usra.edu/meetings/mercury01/pdf/8061.pdf

Prentice, A. J. R., \& Dyt, C.P. 2003, MNRAS, 341, 644

Siegfried, R.W., \& Solomon, S.C. 1974, Icarus, 23, 192 